\title{
Kerr-de Sitter Spacetimes in Various Dimensions and dS/CFT Correspondence
}

\author{
M. H. Dehghani \\ Physics Department and Biruni Observatory, Shiraz University, Shiraz 71454, Iran \\ We consider the Kerr-de Sitter (Kerr-dS) black hole in various dimensions. Intro- \\ ducing a counterterm, we show that the total action of these spacetimes are finite. \\ We compute the masses and the angular momenta of Kerr-dS spaces with one ro- \\ tational parameter in four, five and seven dimensions. These conserved charges are \\ also computed for the case of Kerr-dS space with two rotational parameters in five \\ dimensions. Although the angular momentum density due to the counterterm is \\ nonzero, it gives a vanishing contribution to the total angular momentum. We also \\ find that the total angular momentum of the spacetime is independent of the radius \\ of the boundary for all cases, a fact that is not true for the total mass of the system.
}

\section{INTRODUCTION}

Spacetimes which are asymptotically de Sitter (dS) have attracted a great deal of attention recently [1, 2, 3, 4, 5, 6, 7]. First, this is due to the recent astrophysical data indicating a positive cosmological constant [8], and second because of the understanding of the role of de Sitter spacetimes in string theory and clarification of the microscopic origin of the entropy of dS spacetimes. For AdS spacetimes an anti-de Sitter conformal field theory (AdS/CFT) correspondence is known which posits a relationship between supergravity or string theory in bulk AdS spacetimes and conformal field theory on the boundary [9, 10, 11, 12]. But, for de Sitter spaces, such a relation between the bulk dS and conformal field theory on the boundary is not yet known [5, 7].

The concepts of action and energy momentum play central roles in gravity, but, as is known, there is no good local notion of energy for a gravitating system. A quasilocal definition of the energy and conserved quantities can be found in [13]. The known obstacle to the straightforward definition of the gravitational action and therefore the conserved quantities of a gravitating system is that the action and therefore all the conserved quantities 
diverge. One approach toward evaluating them has been to carry out all computations relative to some other spacetime which is regarded as the ground state for the class of spacetimes of interest. This is done by taking the original action $I_{G}=I_{v}+I_{b}$ for gravity fields and subtracting from it a reference action $I_{0}$, which is a functional of the induced metric $\gamma$ on the boundary $\partial \mathcal{M}$. Conserved quantities are then computed relative to this boundary, which can then be taken to (spatial) infinity if desired.

This approach has been widely successful in providing the conserved quantities of the system in regions of both finite and infinite spatial extent [13, 14]. Unfortunately, it suffers from several drawbacks. The choice of reference spacetime is not always unique [15], nor is it always possible to embed a boundary with a given induced metric into the reference background. Indeed, for Kerr spacetimes this latter problem forms a serious obstruction towards calculating the subtraction energy, and calculations have only been performed in the slow-rotating regime [16]. An extension of this approach was developed for asymptotically AdS spacetimes based on the conjectured AdS /CFT correspondence [17, 18, 19, 20]. Since quantum field theories in general contain counterterms, it is natural from the AdS/CFT viewpoint to append a boundary term $I_{c t}$ to the action which depends on the intrinsic geometry of the (timelike) boundary at large spatial distances. This requirement, along with general covariance, implies that these terms should be functionals of curvature invariants of the induced metric and have no dependence on the extrinsic curvature of the boundary. An algorithmic procedure [21] exists for constructing $I_{c t}$ for asymptotically AdS spacetimes, and so its determination is unique. Addition of $I_{c t}$ will not affect the bulk equations of motion, thereby eliminating the need to embed the given geometry in a reference spacetime. Hence conserved quantities can now be calculated intrinsically for any given spacetime. The efficacy of this approach has been demonstrated in a broad range of examples, all of them in the spatially infinite limit, where the AdS/CFT correspondence applies [22, 23, 24, 25, 26, 27.

Recently, these ideas have been extended to the case of asymptotically de Sitter spacetimes 四, 坷. The purpose of this paper is to investigate the effects of including $I_{c t}$ for Kerr-dS spacetimes in four and higher dimensions. There are several reasons for considering this. Although untill now there is no well defined dS/CFT correspondence, from a gravitational viewpoint the inclusion of additional boundary functionals is not uniquely dependent upon this correspondence and can be carried out even if the dS/CFT correspondence is found to be invalid. It is therefore of interest to explore the implications of $I_{c t}$ for more spacetimes. 
Furthermore, adding the counterterm would change the value of conserved quantities of a finite gravitating system, and thus for consideration of a finite gravitating system the inclusion of $I_{c t}$ is also important. In this paper we extend the idea of using the counterterm introduced by 国, 司 for more than five dimensions. We specifically introduce a counterterm for the class of Kerr-dS black holes in four to seven dimensions. With their lower degree of symmetry relative to spherically symmetric black holes, these spacetimes allow for a more detailed study of the consequences of including $I_{c t}$.

The outline of our paper is as follows. In Sec. II we review the basic formalism and introduce the counterterm. Section III will be devoted to the consideration of Kerr-dS metric with one rotational parameter in various dimensions, and we compute the conserved quantities of these metrics. The conserved charges of the general Kerr-dS spacetimes with two rotational parameters will be computed in the next section. We finish our paper with some concluding remarks.

\section{STRESS TENSOR AND CONSERVED QUANTITIES}

The gravitational action of $(n+1)$-dimensional de Sitter spacetimes is the sum of two terms. The first term is the Einstein-Hilbert volume (or bulk) term with positive cosmological constant $\Lambda=n(n+1) /\left(2 l^{2}\right)$,

$$
I_{v}=-\frac{1}{16 \pi} \int_{\mathcal{M}} d^{n+1} x \sqrt{-g}\left(\mathcal{R}-\frac{2 n(n-1)}{l^{2}}\right),
$$

and the second term is the Gibbons-Hawking boundary term:

$$
I_{b}=\frac{1}{8 \pi} \int_{\partial \mathcal{M}} d^{n} x \sqrt{-\gamma} \Theta(\gamma)
$$

which is chosen such that the variational principle is well defined. The Euclidean manifold $\mathcal{M}$ has metric $g_{\mu \nu}$, covariant derivative $\nabla_{\mu}$, and time coordinate $\tau$ which foliates $\mathcal{M}$ into nonsingular hypersurfaces $\Sigma_{\tau}$ with unit normal $u_{\mu}$ over a real line interval $\Upsilon$. $\Theta$ is the trace of the extrinsic curvature $\Theta^{\mu \nu}$ of any boundary(ies) $\partial \mathcal{M}$ of the manifold $\mathcal{M}$, with induced metric(s) $\gamma_{i j}$. In general $I_{v}$ and $I_{b}$ of Eqs. (1) and (2) are both divergent when evaluated on solutions, as is the Hamiltonian, and other associated conserved quantities [13, 14]. Rather than eliminating these divergences by incorporating a reference term in the spacetime [14, 28], a new term $I_{c t}$ is added to the action which is a functional only of boundary curvature 
invariants. Although there may exist a very large number of possible invariants, one can add in a given dimension only a finite number of them. For an asymptotically AdS spacetime, these can be determined by an algorithmic procedure [21]. These counterterms can be generalized to the case of asymptotically dS spacetimes as

$$
\begin{aligned}
I_{c t}= & \frac{1}{8 \pi} \int_{\partial \mathcal{M}_{\infty}} d^{n} x \sqrt{-\gamma}\left\{\frac{n-1}{l}-\frac{l R}{2(n-2)}-\frac{l^{3}}{2(n-4)(n-2)^{2}}\left[R_{a b} R^{a b}-\frac{n}{4(n-1)} R^{2}\right]\right. \\
& +\frac{l^{5}}{(n-6)(n-4)(n-2)^{3}}\left[\frac{(3 n-2) R}{4(n-1)} R_{a b} R^{a b}-\frac{n(n+2)}{16(n-1)^{2}} R^{3}-2 R^{a c} R_{a b c d} R^{b d}+\right. \\
& \left.\left.\frac{n-2}{2(n-1)} R^{a b} \nabla_{a} \nabla_{b} R-R^{a b} \nabla^{2} R+\frac{1}{2(n-1)} R \nabla^{2} R\right]+\ldots\right\},
\end{aligned}
$$

where $R, R_{a b c d}$ and $R_{a b}$ are the Ricci scalar and Riemann and Ricci tensor of the boundary metric $\gamma_{a b}$.

Now in the following sections we study the implications of including the counterterm for the class of Kerr-dS metrics in various dimensions. As we will show in these cases for a suitable choice of the parameter $m$ we have the inner, outer, and cosmological horizons with radius $r_{-}, r_{+}$, and $r_{c}$. The trace of the extrinsic curvature, $\Theta$ and the square root of the determinant of the metric, $\sqrt{-\gamma}$, are real for $r_{+}<r<r_{c}$ and imaginary for $r>r_{c}$. We will show that the total action

$$
I=I_{v}+I_{b}+I_{c t}
$$

will be finite for Kerr-dS spacetime in various dimensions for the boundary at infinity. Of course one should note that, since for $r>r_{c}$ the counterterm $I_{c t}$ is imaginary, it is necessary to divide $I_{c t}$ by $i$ and then add it to the gravitational action $I_{v}+I_{b}$ to get the total action.

Under the variation of the metric, one obtains

$\delta I=[\text { terms that vanish when the equations of motion hold }]^{\mu \nu} \delta g_{\mu \nu}+\int_{\partial \mathcal{M}} d^{3} x\left(P^{a b}+Q^{a b}\right) \delta \gamma_{a b}$

where $P^{a b}$ is related to the variation of the boundary term:

$$
P^{a b}=\frac{1}{8 \pi}\left(\Theta^{a b}-\Theta \gamma^{a b}\right)
$$

and $Q^{a b}$ is due to the variation of the counterterm (3) given as

$$
\begin{aligned}
Q^{a b}= & \frac{1}{8 \pi}\left\{-\frac{n-1}{l} \gamma^{a b}+\frac{l}{n-2}\left(R^{a b}-\frac{1}{2} R \gamma^{a b}\right)+\frac{l^{3}}{(n-4)(n-2)^{2}}\left[-\frac{1}{2} \gamma^{a b}\left(R^{c d} R_{c d}-\frac{n}{4(n-1)} R^{2}\right.\right.\right. \\
& \left.\left.\frac{n R}{2(n-2)} R^{a b}+2 R_{c d} R^{a c b d}-\frac{n-2}{2(n-1)} \nabla^{a} \nabla^{b} R+\nabla^{2} R^{a b}-\frac{1}{2(n-1)} \gamma^{a b} \nabla^{2} R\right]+\ldots\right\} .
\end{aligned}
$$


One could decompose the metric $\gamma_{a b}$ in the form

$$
\gamma_{a b} d x^{a} d x^{a}=-N^{2} d t^{2}+\sigma_{i j}\left(d \phi^{i}+V^{i}\right)\left(d \phi^{j}+V^{j}\right)
$$

where the coordinates $\phi^{i}$ are the angular variables parametrizing the closed hypersurface of constant $r$ around the origin. The conserved quantities associated with the stress tensors of Eqs. (6) and (7) can be written as

$$
\begin{aligned}
& \mathcal{Q}_{1}(\xi)=\int_{\mathcal{B}} d^{n-1} \phi \sqrt{\sigma} P_{a b} n^{a} \xi^{b}, \\
& \mathcal{Q}_{2}(\xi)=\int_{\mathcal{B}} d^{n-1} \phi \sqrt{\sigma} Q_{a b} n^{a} \xi^{b},
\end{aligned}
$$

where $\sigma$ is the determinant of the metric $\sigma=\gamma_{a b}-n_{a} n_{b}$, and $\xi$ and $n^{a}$ is the Killing vector field and the unit normal vector on the boundary $\mathcal{T}$. As was mentioned both the conserved quantities of Eqs. (9) are infinite, but a linear combination of them is finite. Again, one should note that for the class of Kerr-dS metrics in various dimensions the unit normal $n^{a}$ is timelike for $r_{+}<r<r_{c}$ and spacelike for $r>r_{c}$.

For each Killing vector $\xi$, there exists an associated conserved charge. Thus for our case (Kerr-dS spacetimes in various dimensions) with Killing vector $(\xi=\partial / \partial t)$ and rotational Killing vector $(\varsigma=\partial / \partial \phi)$ we obtain

$$
\begin{aligned}
M & =\int_{\mathcal{B}} d^{n-1} \phi \sqrt{\sigma}\left(P_{a b}+Q_{a b}\right) n^{a} \xi^{b}, \\
J & =\int_{\mathcal{B}} d^{n-1} \phi \sqrt{\sigma}\left(P_{a b}+Q_{a b}\right) n^{a} \varsigma^{b} .
\end{aligned}
$$

These quantities are, respectively, the conserved mass and angular momentum of the system enclosed by the boundary. Note that they may both be dependent upon the location of the boundary $\mathcal{B}$ in the spacetime, although each is independent of the particular choice of foliation $\mathcal{B}$ within the surface $\mathcal{T}$. We find that the different components of the angular momentum are independent of the radius of the boundary for the class of Kerr-dS spaces.

In the context of the $\mathrm{dS} / \mathrm{CFT}$ correspondence the limit in which the boundary $\mathcal{B}$ becomes infinite is taken, and the counterterm prescription ensures that the total action (田) and the conserved charges (10) are finite. No embedding of the surface $\mathcal{T}$ into a reference spacetime is required. This is of particular advantage for the class of Kerr spacetimes, in which it is not possible to embed an arbitrary boundary surface into a flat (or constant-curvature) spacetime [16, 20]. 


\section{KERR-DS METRIC WITH ONE ROTATIONAL PARAMETER}

In this section we consider the class of Kerr-dS family of solutions in various dimensions with one rotational parameter, which can be written as

$$
\begin{aligned}
d s^{2}= & -\frac{\Delta_{r}}{\rho^{2}}\left(d t-\frac{a}{\Xi} \sin ^{2} \theta d \phi\right)^{2}+\frac{\rho^{2}}{\Delta_{r}} d r^{2}+\frac{\rho^{2}}{\Delta_{\theta}} d \theta^{2} \\
& +\frac{\Delta_{\theta} \sin ^{2} \theta}{\rho^{2}}\left[a d t-\frac{\left(r^{2}+a^{2}\right)}{\Xi} d \phi\right]^{2}+r^{2} \cos ^{2} \theta d \Omega_{n-3}
\end{aligned}
$$

in $(n+1)$ dimensions, where

$$
\begin{aligned}
\Delta_{r} & =\left(r^{2}+a^{2}\right)\left(1-\frac{r^{2}}{l^{2}}\right)-2 m r^{4-n}, \\
\Delta_{\theta} & =1+\frac{a^{2}}{l^{2}} \cos ^{2} \theta, \\
\Xi & =1+\frac{a^{2}}{l^{2}} \\
\rho^{2} & =r^{2}+a^{2} \cos ^{2} \theta .
\end{aligned}
$$

\section{A. Kerr-dS $\mathbf{S}_{4}$ metric:}

The metric of Eqs. (11]) and (12) for $n=3$ has three horizons located at $r_{ \pm}$and $r_{c}$, provided the parameter $m$ lies in the range $m_{1, \text { crit }} \leq m \leq m_{2, \text { crit }}$ where $m_{1, \text { crit }}$ and $m_{2, \text { crit }}$ are the two critical masses given by

$$
\begin{aligned}
& m_{1, \text { crit }} \equiv \frac{l}{3 \sqrt{6}} \sqrt{1+33 \frac{a^{2}}{l^{2}}\left(1-\frac{a^{2}}{l^{2}}\right)-\frac{a^{6}}{l^{6}}-\left(1-14 \frac{a^{2}}{l^{2}}+\frac{a^{4}}{l^{4}}\right)^{3 / 2}}, \\
& m_{2, \text { crit }} \equiv \frac{l}{3 \sqrt{6}} \sqrt{1+33 \frac{a^{2}}{l^{2}}\left(1-\frac{a^{2}}{l^{2}}\right)-\frac{a^{6}}{l^{6}}+\left(1-14 \frac{a^{2}}{l^{2}}+\frac{a^{4}}{l^{4}}\right)^{3 / 2}} .
\end{aligned}
$$

It is worthwhile to mention that in the limit $l \rightarrow \infty, m_{1, \text { crit }}=a, m_{2, \text { crit }} \rightarrow \infty, r_{ \pm}=$ $m \pm \sqrt{m^{2}-a^{2}}$, and $r_{c} \rightarrow \infty$ as one may expect. For $m=0$ the metric (11) and (12) is that of pure $\mathrm{dS}_{4}$ spacetime (or flat spacetime if $l \rightarrow \infty$ ), and for $a=0$ the metric is that of Schwarzschild-dS 4 spacetime which has zero angular momentum. Hence we expect the parameters $m$ and $a$ to be associated with the mass and angular momentum of the spacetime respectively. As Eqs. (13) show the critical masses for a Schwarzschild black-hole are 0 and $l / 3 \sqrt{3}$ [4].

Using Eqs. (4), one could show that the total action is finite. The total mass $M$ and the total angular momentum $J_{\phi}$ calculated from Eqs. (10) are given by 


$$
\begin{aligned}
& M=-\frac{m}{\Xi}, \\
& J_{\phi}=\frac{m a}{\Xi^{2}} .
\end{aligned}
$$

\section{B. Kerr-dS $_{5}$ metric}

For the case of $n=4$ the metric given by Eqs. (11) and (12) has an outer and a cosmological horizon provided the parameter $m$ lies in the range $m_{1, \text { crit }} \leq m \leq m_{2, \text { crit }}$ where $m_{1, \text { crit }}$ and $m_{2, \text { crit }}$ are the two critical masses given by

$$
\begin{aligned}
& m_{1, \text { crit }} \equiv \frac{1}{2} a^{2}, \\
& m_{2, \text { crit }} \equiv \frac{l^{2}}{8}\left(1+\frac{a^{2}}{l^{2}}\right) .
\end{aligned}
$$

For $m=0$ the metric (11) and (12) is that of pure $\mathrm{dS}_{5}$ spacetime (or flat spacetime if $l \rightarrow \infty)$, and for $a=0$ the metric is that of Schwarzschild- $\mathrm{dS}_{5}$ spacetime which has zero angular momentum. In this case the critical masses are 0 and $l^{2} / 8$ [ [4]. Again the parameters $m$ and $a$ are associated with the mass and angular momentum of the spacetime, respectively.

Using Eqs. (四), one can show that the total action is finite. The total mass $M$ and the total angular momentum $J_{\phi}$ calculated from Eqs. (10) are given by

$$
\begin{aligned}
M & =-\frac{3 \pi}{4} \frac{m}{\Xi}+\frac{\pi l^{2}}{96}\left[7+\frac{1}{\Xi}\left(\Xi^{2}+1\right)\right], \\
J_{\phi} & =\frac{\pi}{2} \frac{m a}{\Xi^{2}} .
\end{aligned}
$$

\section{Kerr-dS 7 metric:}

The Kerr- $\mathrm{dS}_{7}$ metric with one rotational parameter given by Eqs. (11) and (12) has two horizons located at $r_{+}$and $r_{c}$, provided the parameter $m$ is sufficiently large relative to the other parameters, specifically,

$$
m \geq m_{\text {crit }} \equiv \frac{l^{4}}{27}\left\{1+\frac{3}{2} \frac{a^{2}}{l^{2}}\left(1-\frac{a^{2}}{l^{2}}\right)-\frac{a^{6}}{l^{6}}+\left(1+\frac{a^{2}}{l^{2}}+\frac{a^{4}}{l^{4}}\right)^{3 / 2}\right\} .
$$

For $m=0$ the metric (11) and 12) is that of pure $\mathrm{dS}_{7}$ spacetime (or flat spacetime if $l \rightarrow \infty)$, and for $a=0$ the metric is that of Schwarzschild- $\mathrm{dS}_{7}$ spacetime which has zero 
angular momentum, and the critical mass is $2 l^{4} / 27$. Again parameters $m$ and $a$ should be associated with the mass and angular momentum of the spacetime respectively.

Using Eqs. (4), one can show that the total action is finite. Again the total mass $M$ and the total angular momentum $J_{\phi}$ can be calculated from Eqs. (10) as:

$$
\begin{aligned}
& M=-\frac{5 \pi^{2}}{8} \frac{m}{\Xi}+\frac{\pi^{2} l^{4}}{1280}\left[50-\left(1-\frac{6}{\Xi}\right)(\Xi-1)^{2}\right], \\
& J_{\phi}=\frac{\pi^{2}}{4} \frac{m a}{\Xi^{2}} .
\end{aligned}
$$

Remarkably, we find that the counterterm $Q_{a b} n^{a} \varsigma^{b}$, while non-zero, gives a vanishing contribution upon integration in Eq. (10) for various dimensions of Kerr-dS metrics considered in this section. Also one should note that the total angular momentum $J_{\phi}$ for all the above cases is independent of the radius of the boundary. This was also true for the case of Kerr-AdS spacetime discussed in [20].

\section{THE GENERAL KERR-DS METRIC IN FIVE DIMENSION}

It is known that the number of independent rotational parameters for a metric with rotation group $S O(n)$ is the integer part of $n / 2$. Thus, the metric of the Kerr- $\mathrm{dS}_{5}$ spacetime can have at most two rotational parameters. The metric can be written as

$$
\begin{aligned}
d s^{2}= & -\frac{\Delta_{r}}{\rho^{2}}\left(d t-\frac{a \sin ^{2} \theta}{\Xi_{a}} d \phi-\frac{a \cos ^{2} \theta}{\Xi_{a}} d \psi\right)^{2}+\frac{\rho^{2}}{\Delta_{\theta}} d \theta^{2}+\frac{\rho^{2}}{\Delta_{r}} d r^{2} \\
& +\frac{1-r^{2} / l^{2}}{r^{2} \rho^{2}}\left[a b d t-\frac{b\left(r^{2}+a^{2}\right) \sin ^{2} \theta}{\Xi_{a}} d \phi-\frac{a\left(r^{2}+b^{2}\right) \cos ^{2} \theta}{\Xi_{b}} d \psi\right]^{2} \\
& +\frac{\Delta_{\theta} \sin ^{2} \theta}{\rho^{2}}\left(a d t-\frac{r^{2}+a^{2}}{\Xi_{a}} d \phi\right)+\frac{\Delta_{\theta} \cos ^{2} \theta}{\rho^{2}}\left(b d t-\frac{r^{2}+b^{2}}{\Xi_{b}} d \phi\right),
\end{aligned}
$$

where

$$
\begin{aligned}
& \Delta_{r}=\frac{1}{r^{2}}\left(r^{2}+a^{2}\right)\left(r^{2}+b^{2}\right)\left(1-\frac{r^{2}}{l^{2}}\right)-2 m \\
& \Delta_{\theta}=1+\frac{a^{2}}{l^{2}} \cos ^{2} \theta+\frac{b^{2}}{l^{2}} \sin ^{2} \theta \\
& \Xi_{a}=1+\frac{a^{2}}{l^{2}}, \quad \Xi_{b}=1+\frac{b^{2}}{l^{2}} \\
& \rho^{2}=r^{2}+a^{2} \cos ^{2} \theta+b^{2} \sin ^{2} \theta .
\end{aligned}
$$


Again this metric has two event and a cosmological horizon provided the parameter $m$ lies between the two critical masses given by Eqs. (15). Using Eqs. (四) and Eqs. (10) one can show that the total action is finite and the total mass $M$ and the total angular momenta $J_{\phi}$ and $J_{\psi}$ are

$$
\begin{aligned}
& M=-\frac{3 \pi m}{4 \Xi_{a} \Xi_{b}}+\frac{\pi l^{2}}{96}\left[7+\frac{\left(\Xi_{a}^{2}+\Xi_{b}^{2}\right)}{\Xi_{a} \Xi_{b}}\right], \\
& J_{\phi}=\frac{\pi}{2 \Xi_{a}^{2} \Xi_{b}} m a, \quad J_{\psi}=\frac{\pi}{2 \Xi_{a} \Xi_{b}^{2}} m a .
\end{aligned}
$$

Again it is worthwhile to mention that the $\phi$ and $\psi$ components of the angular momentum due to the counterterm is zero, and the total angular momenta $J_{\phi}$ and $J_{\psi}$ are independent of the radius of the boundary.

\section{CLOSING REMARKS}

In this paper we have computed the conserved charges of Kerr-dS spacetimes in various dimensions through the use of the Brown-York boundary stress tensor. Of course this is the first step in studying the prospects for a duality between quantum gravity on de Sitter space and a Euclidean field theory defined on the boundary.

By introducing a counterterm, $I_{c t}$ we showed that the total action will be finite as the boundary goes to infinity. Although, the angular momentum densities due to the counterterm for various dimensions are not zero, they give vanishing contributions upon integration. Thus, the total angular momenta of the spacetimes for various dimensions are due only to the boundary terms which are independent of the radius of the boundary, a fact that is not true for the total mass of the spacetime. As in the case of anti-de Sitter spaces the total mass in odd dimensions has a term which is proportional to the parameter $m$, and a second term which is due to the de Sitter spaces.

[1] A. Strominger, J. High Energy Phys. 10, 034 (2001).

[2] V. Balasubramanian, P. Horova, and D. Minic, J. High Energy Phys. 05, 043 (2001).

[3] E. Witten, "Quantum gravity in de Sitter space", hep-th/0106109.

[4] D. Klemm, Nucl. Phys. B625, 295 (2002). 
[5] V. Balasubramanian, J. D. Boer, and D. Minic, "Mass, Eentropy and Holography in Asymptotic de Sitter Spaces", hep-th/0110108; A. M. Ghezelbash and R. B. Mann, J. High Energy Phys. 01, 005 (2002).

[6] M Spradlin, A. Strominger, and A. Volovich, "Les Houches Lectures on the de Sitter spaces", hep-th/0110007.

[7] D. Klemm, Class. Quantum Grav. 19, 579 (2002); S. Nojiri and S. D. Odintsov, Phys. Lett. B 519, 145 (2001); 523, 165 (2001); S. Nojiri, S. D. Odintsov and S. Ogushi, Phys. Rev. D 65, 023521 (2002); T. Shiromizu, D. Ida and T. Torii, J. High Energy Phys. 11, 010 (2001); R. G. Cai, Y. S. Myung and Y. Z. Zhang, Phys. Rev. D (to be published), hep-th/0110234.

[8] S. Perlmutter, in Proceeding of the 19th Internatinal Symposium on Photon and Lepton Interaction at High Energy Physics LP99, edited by J. A. Jaros and M. E. Peskin [Int. J. Mod. Phys. A 15, 715 (2000)], e CONFEC 990809, 717 (2000).

[9] E. Witten, Adv. Theor. Math. Phys. 2, 253 (1998).

[10] S. W. Hawking, J. Maldacena, A. Strominger, J. High Energy Phys. 05, 001 (2001).

[11] O. Aharony, S. S. Gubser, J. Maldacena, H. Ooguri and Y. Oz, Phys. Rept., 323, 183 (2000).

[12] M. H. Dehghani, A. M. Ghezelbash and R. B. Mann, Nucl. Phys. B625, 389 (2002).

[13] J. D. Brown and J. W. York, Phys. Rev. D 47, 1407 (1993).

[14] J. D. Brown, J. Creighton and R.B. Mann, Phys. Rev. D 50, 6394 (1994).

[15] K. C. K. Chan, J.D.E. Creighton, and R. B. Mann Phys. Rev. D 54, 3892 (1996).

[16] E. A. Martinez, Phys. Rev. D 50, 4920 (1994).

[17] M. Hennigson and K. Skenderis, J. High Energy Phys. 07, 023 (1998).

[18] S. Hyun, W. T. Kim and J. Lee, Phys. Rev. D 59, 084020 (1999).

[19] V. Balasubramanian and P. Kraus, Commun. Math. Phys. 208, 413 (1999).

[20] M. H. Dehghani and R. B. Mann, Phys. Rev. D 64, 044003 (2001).

[21] P. Kraus, F. Larsen and R. Siebelink, Nucl. Phys. B 563, 259 (1999).

[22] R. B. Mann, Phys. Rev. D 60, 104047 (1999).

[23] R. Emparan, C. V. Johnson and R. C. Myers, Phys. Rev. D 60, 104001 (1999).

[24] R. B. Mann, Phys. Rev. D 61, 084013 (2000).

[25] S. Das and R. B. Mann, J. High Energy Phys. 08, 033 (2000).

[26] A. M. Awad and C. V. Johnson, Phys. Rev. D 61, 084025 (2000).

[27] A. DeBenedictus and K. Vishwanathan, hep-th/9911060. 
[28] I. S. Booth and R.B. Mann, Phys. Rev. D 59, 064021 (1999). 